\title{
Associação entre impactos funcionais e psicossociais das desordens bucais e qualidade de vida entre idosos
}

\author{
Association between physical and psychosocial impacts \\ of oral disorders and quality of life among the elderly
}

Andréa Maria Eleutério de Barros Lima Martins ${ }^{1}$

Kimberly Marie Jones ${ }^{2}$

João Gabriel Silva Souza ${ }^{3}$

Isabela Almeida Pordeus ${ }^{4}$

${ }^{1}$ Departamento de Odontologia, Centro de

Ciências Básicas e da Saúde, Universidade Estadual de

Montes Claros. Av. Rui Braga SN, Vila Mauricéia. 39.401-089 Montes Claros MG Brasil. martins. andreamebl@gmail.com

${ }^{2}$ Faculdades Unidas do

Norte de Minas.

${ }^{3}$ Faculdade de Odontologia de Piracicaba, Universidade

Estadual de Campinas.

${ }^{4}$ Departamento de

Odontopediatria,

Faculdade de Odontologia,

Universidade Federal de

Minas Gerais.
Abstract This study sought to evaluate the association between the impact of oral disorders in terms of physical/psychosocial dimensions and quality of life among the elderly. It involved a cross-sectional study conducted among the elderly (65-74 years) in 2008/2009. The social impact was assessed using the Oral Health Impact Profile (OHIP 14) and the quality of life using the SF 12 Short-Form Health Survey. Descriptive, univariate and multivariate (logistic regression) analysis was conducted with correction for the design effect, using SPSS ${ }^{\circledR} 18.0$ software. Of the 800 individuals approached, 736 elderly individuals participated ( $T R=92 \%)$, with a mean age of 67.77 years, the majority of whom showed no impact based on the measurement of the prevalence of OHIP. The functional limitation dimension of the OHIP was associated with the physical domain of the SF12, irrespective of the other variables investigated. However, the seriousness of OHIP and its psychological discomfort and disability dimensions was associated with the mental domain of the SF12. The conclusion reached is that some impacts of oral disorders were associated with unsatisfactory quality of life in the physical and mental domains.

Key words Quality of Life, Elderly individuals, Oral health, Health
Resumo Objetivou-se avaliar a associação entre o impacto das desordens bucais em suas dimensões fisica/psicossocial a a qualidade de vida entre idosos. Estudo transversal conduzido entre idosos (65-74 anos), em 2008/2009. O impacto foi avaliado através do Oral Health Impact Profile (OHIP 14) e a qualidade de vida, através do Short-Form Health Survey SF 12. Foram realizadas análises descritivas, univariadas e múltiplas (regressões logísticas), com correção pelo efeito de desenho, utilizando-se o PASW ${ }^{\circledR}$ 18.0. Dos 800 convidados, participaram 736 idosos (TR $=92 \%)$, com a média de idade de 67,77 anos, a maioria não apresentou impacto, a partir da medida da prevalência do OHIP. A dimensão limitação funcional do OHIP foi associada ao dominio físico do SF12, independentemente de outras variáveis investigadas. Já a gravidade do OHIP, as suas dimensões de desconforto psicológico e deficiência, foram associadas ao dominio mental do SF12. Conclui-se que alguns impactos das desordens bucais estiveram associados a uma qualidade de vida insatisfatória em seus domínios físico e mental.

Palavras-chave Qualidade de vida, Idoso, Saúde bucal, Saúde 


\section{Introdução}

A diminuição nas taxas de mortalidade, o declínio nas taxas de natalidade e a elevação da expectativa de vida devido às melhorias nos cuidados e nas políticas públicas de saúde contribuíram para o aumento da proporção de idosos no mundo ${ }^{1,2}$. Essa transição demográfica gerou entre os idosos uma carga crescente de doenças com impacto negativo na sua qualidade de vida ${ }^{1}$, definida pela Organização Mundial de Saúde (OMS) como a percepção do indivíduo de sua posição na vida, no contexto da cultura e sistema de valores nos quais ele vive e em relação aos seus objetivos, expectativas, padrões e preocupações ${ }^{3}$. A qualidade de vida avalia o impacto da doença e do tratamento na capacidade da pessoa para realizar atividades diárias, reconhece o valor da saúde de forma ampla, considerando a avaliação de outros fatores além do desconforto ou dor, trata-se de um conceito multiatributo que engloba diversas dimensões físicas, mentais ou funcionais, bem-estar psicológico e social ${ }^{1,4,5}$. A avaliação da qualidade de vida exige que os profissionais de saúde repudiem um foco estrito da doença para uma abordagem mais ampla, ou seja, psicossocial. Investigações prévias buscaram identificar a relação entre o impacto da saúde geral $^{6,7}$ e da saúde bucal ${ }^{8,9}$ na qualidade de vida.

A saúde bucal é parte da saúde geral e essencial para a qualidade de vida ${ }^{10,11}$. O julgamento da saúde bucal tem sido conduzido a partir da avaliação centrada nas condições dentárias e/ ou bucais denominadas condições objetivas ou normativas (conduzidas por cirurgiões dentistas, conforme normas pré-estabelecidas) ${ }^{12}$. As condições normativas por si só não indicam o quanto a pessoa se sente afetada pela sua condição bu$\mathrm{cal}^{13}$. Nesse contexto, alguns instrumentos foram desenvolvidos para avaliar questões subjetivas da saúde bucal ${ }^{14-16}$, entre eles destaca-se o Oral Health Impact Profile (OHIP), em especial em sua forma abreviada, o OHIP- $14^{15}$. O OHIP foi proposto para avaliar as percepções dos indivíduos sobre o impacto das desordens bucais em seu bem-estar, através de questões que abrangem sete dimensões: limitação funcional, dor física, desconforto psicológico, incapacidade física, incapacidade psicológica, incapacidade social e deficiência na realização das atividades cotidianas ${ }^{15}$.

Ele tem sido utilizado para avaliar o impacto da saúde bucal na qualidade de vida ${ }^{14,17-19}$. Segundo Locker e Quiñonez ${ }^{9}$, os impactos capturados pelo OHIP parecem não afetar diretamente a qualidade de vida do indivíduo a qualquer grau apreciável, uma vez que podem não ter magnitude ou duração suficiente para comprometer a qualidade de vida global. No entanto, os impactos consequentes das desordem bucais podem afetar as dimensões físicas e psicossociais da saúde bucal, havendo a possibilidade dessas dimensões comprometerem ou não a qualidade de vida9. Para verificar tal associação, Locker e Quiñonez ${ }^{9}$ avaliaram a qualidade de vida a partir de uma única questão criada por eles mesmos, não sendo identificados estudos que tivessem investigado essa associação a partir da utilização de questionário desenvolvido e validado para avaliar a qualidade de vida. Nessa perspectiva, é recomendada a investigação da existência de associação entre o impacto das desordens bucais em suas dimensões física/psicossocial com os domínios da qualidade de vida a partir de instrumentos desenvolvidos e validados para investigar essas questões entre idosos. Dessa forma, a presente investigação avaliou a possível associação entre o impacto das desordens bucais captadas pelo OHIP- $14^{15} \mathrm{e}$ a qualidade de vida de idosos avaliada a partir do Short-Form Health Survey $(\mathrm{SF}-12)^{20}$.

\section{Método}

Estudo transversal analítico conduzido entre idosos (65-74 anos) de um município brasileiro de grande porte populacional, Montes Claros, Minas Gerais, entre 2008 e 2009 $9^{21-24}$, que utilizou os critérios de avaliação das condições de saúde bucal propostos pela OMS em $1997^{25}$. A amostra estimada foi de 740 dentre 9929 idosos do município avaliado, definida considerando a prevalência estimada em 0,50, nível de confiança de $95 \%$, erro amostral de 5,5\%, deff (design effect) igual a 2,0, uma taxa de não resposta de $20 \%$, para se compensar as possíveis perdas. Essa amostra foi complexa probabilística por conglomerados em dois estágios (setores censitários e quadras), foram selecionados 53 dos 276 setores censitários urbanos e duas das 11 áreas rurais, através de amostragem aleatória simples. Em seguida, também por amostragem aleatória simples, foram selecionadas as quadras em cada um dos 53 setores. Todos os domicílios das quadras selecionadas foram visitados. $\mathrm{Na}$ zona rural, os residentes em todos os domicílios, situados a uma distância de até 500 metros de uma instituição de referência, foram convidados a participar ${ }^{24}$.

A coleta dos dados foi realizada por 24 cirurgiões-dentistas treinados e calibrados (Kappa inter/intraexaminadores e coeficiente de correlação intraclasse) para a realização das entrevistas 
e exames intrabucais, realizados em ambiente amplo, sob iluminação natural, empregando-se espelho clínico bucal e sonda CPI previamente esterilizados $^{22}$. Participaram da coleta de dados examinadores que apresentaram concordância $\geq 0,60$, conforme escala proposta por Fleiss ${ }^{26}$ modificada por Cicchetti et al. ${ }^{27}$. Os dados foram registrados em um programa de computador de mão, empregando-se o Programa Coletor de dados em saúde desenvolvido para essa pesquisa ${ }^{23}$.

As variáveis dependentes do estudo foram os domínios físico e mental da qualidade de vida. Optou-se pelo uso da versão validada no Brasil do 12 - Item Short-Form Health Survey (SF-12) ${ }^{20}$, composto por 12 itens que, considerando a percepção do indivíduo em relação aos aspectos de sua vida nas quatro últimas semanas, avalia $o$ impacto da saúde geral na qualidade de vida nas dimensões da capacidade funcional, dos aspectos físicos, sociais e emocionais, da dor, da vitalidade e da saúde mental ${ }^{20}$. Cada um dos 12 itens possui um conjunto de possíveis respostas distribuídas em uma escala tipo Likert, sendo possível, a partir da aplicação de um algoritmo próprio do questionário, com pontuações padronizadas e normatizadas, produzir escores de $0-100$, onde valores iguais a 50 referem-se à população normal ${ }^{20}$. Uma vez que a saúde e, portanto, a qualidade de vida são influenciadas pelos domínios físico (DF) e mental (DM), estes domínios são analisados em separado, cujos pontos de corte nos escores gerados foram 56,57 e 60,75 , respectivamente, nos quais a qualidade de vida insatisfatória nos domínios referidos foram estimadas por valores superiores a esses pontos de corte $^{28}$.

Para avaliar o impacto das desordens bucais nas suas dimensões física e psicossocial, foi utilizada a versão traduzida e validada para a língua portuguesa e cultura brasileira do OHIP- $14^{29}$, composto por 14 questões. O OHIP-14 foi desenvolvido e permitiu a conservação das dimensões originais do OHIP-49 com boa confiabilidade, validade e precisão, tornando-se útil em estudos em que apenas um número limitado de questões pode ser aplicado ${ }^{15}$. Para cada pergunta do OHIP14 , os idosos foram convidados a responder sobre a situação da sua boca e dos seus dentes nos últimos seis meses. Para a codificação das respostas do OHIP, foi utilizada uma escala do tipo Likert, que aborda a frequência experimentada num impacto, como se segue: 0-nunca, 1-raramente, 2 -às vezes, 3 -repetidamente, 4 -sempre. Para análise e interpretação dos impactos avaliados pelo OHIP-14, foi considerada a proposta de Locker e Quiñonez (2011). A prevalência do impacto foi obtida pelas respostas repetidamente ou sempre a um ou mais itens do OHIP, a gravidade foi aferida pela soma de todos os escores, variando de 0 a 56, sendo que quanto maior o escore, maior é o impacto decorrente das desordens bucais sobre seu bem-estar. Sua extensão foi avaliada pela soma do número de itens com impacto, variando de 0 a 14 , considerando as perguntas respondidas pelas opções repetidamente e sempre? .

As outras variáveis independentes foram referentes às características pessoais, à saúde geral, a comportamentos, a condições normativas e subjetivas de saúde bucal. As variáveis relativas às condições normativas e subjetivas de saúde geral foram presença de doenças crônicas (não, sim), uso de medicamentos (não, sim), versão validada e adaptada à cultura brasileira do Mini Exame do Estado Mental ${ }^{30}$ (sem déficit cognitivo, com déficit cognitivo), atividade física (sempre ou frequentemente, ocasionalmente, raramente ou nunca), satisfação com a vida (satisfeito/muito satisfeito, nem satisfeito e nem insatisfeito, insatisfeito/muito insatisfeito). As características pessoais consideradas foram faixa etária em anos (65-68, 69-74), raça autodeclarada (branco, pardo/amarelo/negro/ indígena), sexo (feminino, masculino), estado civil (casado/união estável, viúvo/divorciado, solteiro), escolaridade em anos de estudo (9 ou mais, 5-8, 0-4), renda per capita (mais de um salário, até um salário).

No grupo de comportamentos foram avaliadas a frequência diária da higiene bucal (mais de 2 vezes, 1 ou 2 vezes, nenhuma vez), hábitos tabagista e etilista atual ou passado (ausente, presente), serviço odontológico utilizado (Sistema Único de Saúde - SUS, outros serviços). Ressalta-se que o SUS é o sistema de saúde brasileiro que é baseado no princípio da saúde como um direito do cidadão e um dever do Estado ${ }^{31}$.

As condições normativas de saúde bucal avaliadas foram alterações da mucosa bucal (não, sim), presença de edentulismo (não, sim), uso de prótese dentária (não, sim), necessidade normativa de tratamento odontológico (não, sim). As condições subjetivas de saúde bucal foram percepção da saúde bucal, da mastigação, da aparência de dentes e gengivas e da fala devido aos dentes e gengivas (ótima/boa, regular, ruim/péssima), percepção do relacionamento devido à condição bucal (não afetado, afetado), percepção da necessidade de tratamento (sim, não); dor de dentes e gengivas nos últimos seis meses (não, sim) e satisfação com o atendimento odontológico (satisfeito, insatisfeito).

Para a análise dos dados, empregou-se o software PASW ${ }^{\circledR}$ Statistics 18.0. Realizou-se a correção 
pelo efeito desenho, já que o estudo baseou-se em amostra complexa por conglomerados em dois estágios. $\mathrm{Na}$ análise descritiva incluiu-se a frequência absoluta (n), a frequência relativa (\%) e a frequência relativa com correção pelo efeito de desenho $(\%$ ), o erro padrão (\%) e o deff para variáveis categóricas. Em seguida, foram conduzidas análises univariadas a partir do Teste Qui-quadrado. Foram incluídas nas análises múltiplas aquelas variáveis que apresentaram nível de significância (valor-p) igual ou inferior a 0,20. Foram feitas regressões logísticas para estimativa dos modelos múltiplos, ficaram retidos nos modelos os fatores que permaneceram associados ao nível de $\mathrm{p} \leq 0,05$, com intervalos de $95 \%$ de confiança. Os princípios éticos desse estudo estiveram de acordo com a Resolução do Conselho Nacional de Saúde-(S) do Brasil, n ${ }^{\circ} 196 / 96^{32}$, sob aprovação do Comitê de Ética em Pesquisa, CEP/Unimontes. Todos os participantes do estudo assinaram o termo de consentimento livre e esclarecido.

\section{Resultados}

Estimou-se a participação de 740 idosos, foram encontrados nos domićlios dos conglomerados sorteados 800 idosos. Destes, 736 (92\%) aceitaram participar da investigação e foram avaliados. Observou-se que a média de idade foi de 67,77 anos $(\mathrm{EP}=0,15)$, a maioria se enquadrava na categoria pardo/amarelo/negro/indígena, dentre os 736, 12 $\left(1,6 \%{ }^{*}\right)$ relatou nunca ter utilizado os serviços odontológicos (Tabela 1).

Identificou-se uma maior prevalência de qualidade de vida insatisfatória no domínio físico e satisfatória no domínio mental do SF 12. Os idosos foram caracterizados quanto às questões do SF 12 (Tabela 2).

$\mathrm{Na}$ avaliação do impacto das desordens bucais nas suas dimensões físicas e psicossociais, avaliada pelo OHIP-14, sua extensão apresentou média de 0,55 , com desvio padrão de $\pm 1,57$ e mediana de 0,00 , com valores que variam de 0 a 14 . A gravidade do impacto obteve média de 6,18 , desvio padrão de $\pm 8,41$ e mediana de 2,00. Identificou-se que a maioria dos idosos investigados não obteve impacto na avaliação pelo OHIP-14, a partir da medida de sua prevalência (Tabela 3 ).

$\mathrm{Na}$ análise univariada constatou-se que variáveis pertencentes ao OHIP, características pessoais, saúde geral, comportamentos e condições normativas e subjetivas de saúde bucal, estiveram estatisticamente associadas $(\leq 0,20)$ aos domínios da qualidade de vida dos idosos (Tabela 4 ).
Nas análises múltiplas constatou-se que quanto ao domínio físico da qualidade de vida houve maior chance de qualidade de vida insatisfatória entre os que relataram limitação funcional (dimensão 1 do OHIP 14), presença de doença crônica, hábito etilista atual ou passado, ter utilizado serviços odontológicos prestados pelo SUS, presença de dor nos dentes nos últimos seis meses e menor chance de qualidade de vida insatisfatória entre os homens. Quanto ao domínio mental houve maior chance de qualidade de vida insatisfatória entre os que relataram maior gravidade do OHIP, desconforto psicológico (dimensão 2 do OHIP 14), deficiência (dimensão 7 do OHIP 14), uso de medicamentos, estar nem satisfeitos e nem insatisfeitos com a vida, estar insatisfeitos ou muito insatisfeitos com a vida (Tabela 5).

\section{Discussão}

Estudo prévio propõe que a utilização do OHIP14 , por si só, não permite o estabelecimento da associação direta entre qualidade de vida e saúde bucal $^{9}$, visto que qualidade de vida é um termo frequentemente usado na literatura científica médica e social, que possui caráter multidimensional e é difícil de ser definido. Em algumas definições, qualidade de vida e saúde são termos indistinguíveis, porém, há um crescente reconhecimento de que a qualidade de vida refere-se a algo muito mais amplo do que a saúde ${ }^{33}$. Na presente investigação foi testada a associação entre os impactos decorrentes das desordens bucais e a qualidade de vida, considerando possíveis fatores de confusão. A qualidade de vida, devido a seu caráter multidimensional, pode ser influenciada por diversos fatores, entre eles características pessoais, socioeconômicas e demográficas ${ }^{34}$, condições normativas ${ }^{35}$ e subjetivas $^{9}$ de saúde.

Foi identificada associação entre o domínio físico da qualidade de vida e a dimensão - limitação funcional - da condição de saúde bucal. A expressão "saúde bucal" aborda um conjunto de condições normativas e subjetivas, que possibilita ao ser humano exercer funções como mastigação, deglutição e fonação, exercitar a autoestima e relacionar-se socialmente. Essas condições devem ser percebidas pelo indivíduo de maneira favorável, uma vez que a saúde bucal é parte integrante da saúde geral e essencial ao bem-estar e à qualidade de vida das pessoas ${ }^{36}$. A cavidade bucal tem sido historicamente dissociada do resto do corpo, quando se considera o estado geral de saúde. Questões funcionais relacionadas à saúde bucal, como 
Tabela 1. Caracterização da população idosa de Montes Claros (MG), Brasil, 2008/2009, quanto às características pessoais, à saúde geral, aos comportamentos e quanto às condições normativas e subjetivas de saúde bucal. $\mathrm{n}=$ 736.

\begin{tabular}{|c|c|c|c|c|c|}
\hline & & & & rropac & \\
\hline & $\mathbf{n}$ & $\%$ & $\%^{\mathrm{a}}$ & $\%$ & Deff \\
\hline Características pessoais & & & & & \\
\hline Faixa etária (em anos) & & & & & \\
\hline $65-68$ & 382 & 51,90 & 52,00 & 2,1 & 1,399 \\
\hline $69-74$ & 354 & 48,10 & 48,00 & & \\
\hline Raça autodeclarada ${ }^{*}$ & & & & & \\
\hline Branco & 246 & 33,60 & 32,60 & 3,8 & 5,416 \\
\hline Pardo/Amarelo/Negro/Indígena & 487 & 66,40 & 67,40 & & \\
\hline Sexo & & & & & \\
\hline Feminino & 423 & 57,50 & 56,50 & 2,3 & 1,778 \\
\hline Masculino & 313 & 42,50 & 43,50 & & \\
\hline Estado Civil & & & & & \\
\hline Casado/União estável & 449 & 61,00 & 64,50 & 3,5 & 4,502 \\
\hline Viúvo/Divorciado & 246 & 33,40 & 30,60 & 3,1 & 3,762 \\
\hline Solteiro & 41 & 5,60 & 4,80 & 1,1 & 2,132 \\
\hline Escolaridade (anos de estudo) & & & & & \\
\hline 9 ou mais & 97 & 13,20 & 12,80 & 2,0 & 3,005 \\
\hline $5-8$ & 141 & 19,20 & 17,20 & 2,3 & 3,038 \\
\hline $0-4$ & 498 & 67,70 & 70,00 & 3,4 & 4,450 \\
\hline Renda per capita em salários mínim & & & & & \\
\hline Mais de 1 & 217 & 30,50 & 29,00 & 3,7 & 5,246 \\
\hline Até 1 & 495 & 69,50 & 71,00 & & \\
\hline Condições normativas e subjetivas de & & & & & \\
\hline Presença de doenças crônicas* & & & & & \\
\hline Não & 115 & 15,70 & 16,80 & 2,4 & 3,229 \\
\hline Sim & 619 & 84,30 & 83,20 & & \\
\hline Uso de medicamentos ${ }^{*}$ & & & & & \\
\hline Não & 197 & 26,90 & 27,20 & 2,0 & 1,653 \\
\hline Sim & 536 & 73,10 & 72,80 & & \\
\hline Minimental (ajustado por escolarid & & & & & \\
\hline Sem déficit cognitivo & 501 & 79,30 & 79,40 & 2,1 & 1,909 \\
\hline Com déficit cognitivo & 131 & 20,70 & 20,60 & & \\
\hline Atividade física* & & & & & \\
\hline Sempre ou frequentemente & 134 & 18,20 & 19,10 & 3,0 & 4,714 \\
\hline Ocasionalmente & 134 & 18,20 & 18,20 & 2,6 & 3,746 \\
\hline Raramente ou nunca & 467 & 63,60 & 62,70 & 4,2 & 6,213 \\
\hline Satisfação com a vida* & & & & & \\
\hline Satisfeito ou muito satisfeito & 605 & 82,50 & 82,50 & 2,2 & 2,819 \\
\hline Nem satisfeito e nem insatisfeito & 103 & 14,00 & 14,30 & 1,9 & 2,428 \\
\hline Insatisfeito ou muito insatisfeito & 26 & 3,50 & 3,10 & 0,9 & 2,032 \\
\hline Comportamentos & & & & & \\
\hline Higiene bucal/Frequência diária* & & & & & \\
\hline Mais de 2 vezes & 324 & 44,70 & 43,00 & 3,7 & 4,497 \\
\hline 1 ou 2 vezes & 378 & 52,10 & 53,20 & 3,6 & 4,226 \\
\hline Nenhuma vez & 23 & 3,20 & 3,80 & 1,4 & 4,648 \\
\hline Hábito tabagista atual ou passado* & & & & & \\
\hline Ausente & 477 & 64,90 & 64,90 & 2,6 & 2,509 \\
\hline Presente & 258 & 35,10 & 35,10 & & \\
\hline Hábito etilista atual ou passado* & & & & & \\
\hline Ausente & 460 & 62,70 & 61,10 & 3,0 & 3,137 \\
\hline Presente & 274 & 37,30 & 38,90 & & \\
\hline Serviço odontológico utilizado* & & & & & \\
\hline SUS & 232 & 32,40 & 34,60 & 4,6 & 7,433 \\
\hline Outros serviços & 485 & 67,60 & 65,40 & & \\
\hline
\end{tabular}




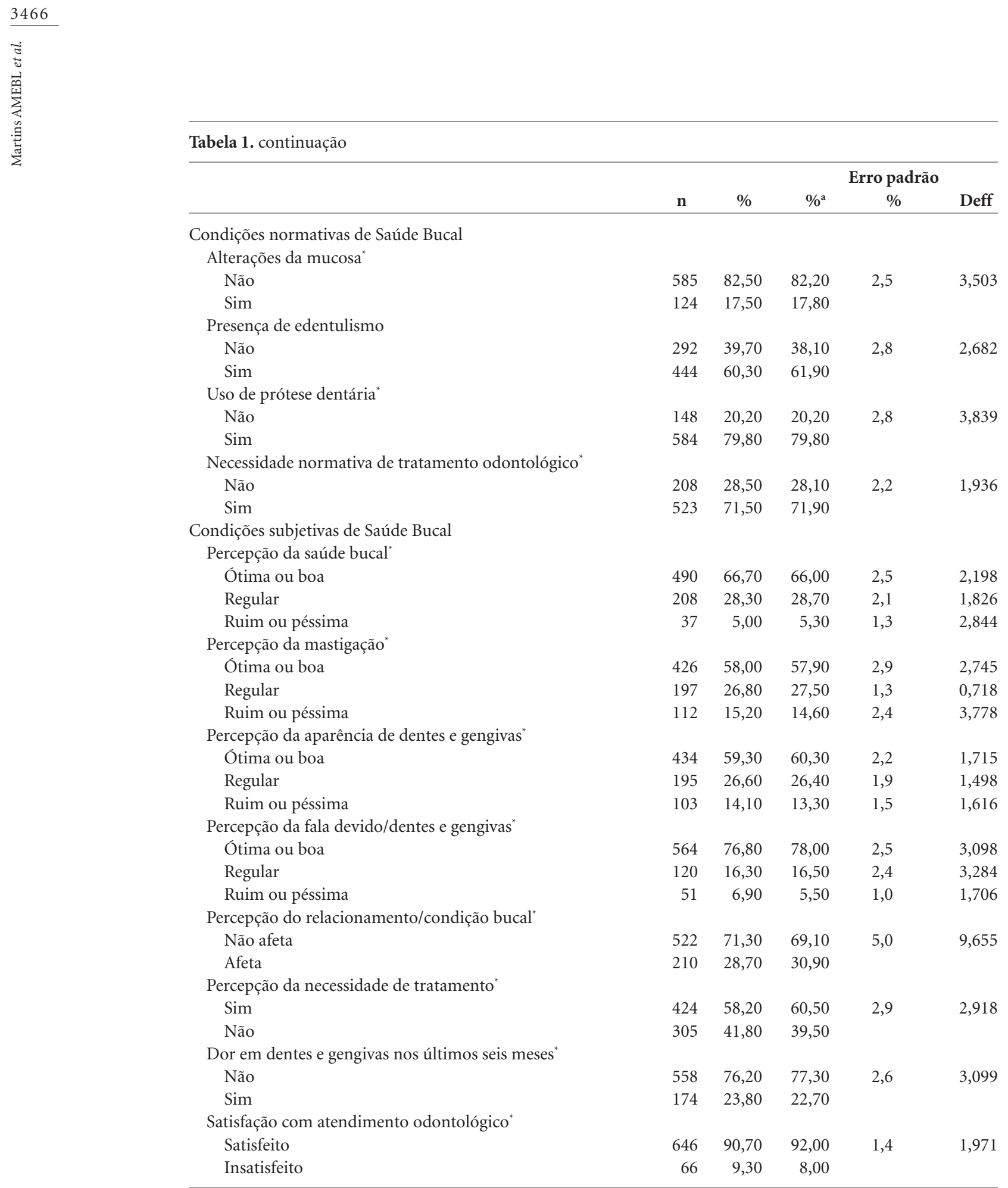

aalores estimados com a correção pelo efeito desenho. ${ }^{\mathrm{b}}$ Baseada no salário mínimo em 2008, no valor de R\$415,00 (Us\$ 173,64).

"Variáveis que apresentaram "n" inferior a 736 devido à perda de informações.

necessidade de próteses dentárias, podem influenciar a associação entre o impacto das desordens bucais e a qualidade vida entre idosos brasileiros ${ }^{16}$. Os idosos brasileiros, assim como os participantes dessa investigação, possuem altas taxas de edentulismo e necessidade de tratamento odontológico ${ }^{37}$, fatores que podem influenciar a mastigação, entre outras limitações funcionais. Uma vez que entre os idosos investigados constatou-se associação entre limitação funcional e domínio físico da qualidade de vida, há necessidade de incrementar a oferta de serviços odontológicos especialmente no âmbito do SUS com o intuito de melhorar a qualidade de vida desses idosos. 
Tabela 2. Distribuição da frequência da população idosa de Montes Claros (MG), quanto às questões do SF-12, $2008 / 2009, \mathrm{n}=736$.

\begin{tabular}{|c|c|c|c|c|c|}
\hline \multirow[t]{2}{*}{ Variáveis } & \multirow[b]{2}{*}{$\mathbf{n}$} & \multirow[b]{2}{*}{$\%$} & \multicolumn{3}{|c|}{ Erro padrão } \\
\hline & & & $\%^{\mathrm{a}}$ & $\%$ & Deff \\
\hline \multicolumn{6}{|l|}{ Qualidade de vida } \\
\hline \multicolumn{6}{|l|}{ Domínio físico do SF12* } \\
\hline Satisfatório & 260 & 35,50 & 35,50 & 3,4 & 4,063 \\
\hline Insatisfatório & 472 & 64,50 & 64,50 & & \\
\hline \multicolumn{6}{|l|}{ Domínio mental do SF $12^{*}$} \\
\hline Satisfatório & 594 & 81,10 & 81,50 & 1,8 & 1,744 \\
\hline Insatisfatório & 138 & 18,90 & 18,50 & & \\
\hline \multicolumn{6}{|c|}{ 1- Em geral, o (a) Sr.(a) diria que a sua saúde é?* } \\
\hline Excelente & 34 & 4,60 & 5,20 & 1,2 & 2,606 \\
\hline Muito Boa & 99 & 13,50 & 15,80 & 3,5 & 7,717 \\
\hline Boa & 221 & 30,10 & 28,90 & 2,1 & 1,768 \\
\hline Regular & 300 & 40,90 & 40,00 & 3,3 & 3,647 \\
\hline Ruim & 80 & 10,90 & 10,10 & 1,2 & 1,234 \\
\hline \multicolumn{6}{|c|}{$\begin{array}{l}2 \text { - O (a) Sr. (a) acha que sua saúde, agora, o dificulta de } \\
\text { fazer algumas coisas do dia a dia, como por exemplo: } \\
\text { atividades médias (como mover uma cadeira, fazer } \\
\text { compras, limpar a casa, trocar de roupa).* }\end{array}$} \\
\hline Sim, muita dificuldade & 117 & 15,90 & 15,00 & 2,1 & 2,922 \\
\hline Sim, pouca dificuldade & 245 & 33,40 & 30,40 & 2,4 & 2,141 \\
\hline Não tive dificuldade & 372 & 50,70 & 54,60 & 3,1 & 3,179 \\
\hline \multicolumn{6}{|c|}{$\begin{array}{l}3 \text { - O (a) Sr. (a) acha que sua saúde, agora, o dificulta de } \\
\text { fazer algumas coisas do dia a dia, como por exemplo: } \\
\text { subir três ou mais degraus de escada.* }\end{array}$} \\
\hline Sim, muita dificuldade & 111 & 15,10 & 13,60 & 1,8 & 2,224 \\
\hline Sim, pouca dificuldade & 249 & 33,90 & 32,00 & 2,6 & 2,452 \\
\hline Não tive dificuldade & 374 & 51,00 & 54,40 & 3,3 & 3,638 \\
\hline \multicolumn{6}{|c|}{$\begin{array}{l}4 \text { - Durante as últimas } 4 \text { semanas, o (a) Sr.(a) teve algum } \\
\text { dos seguintes problemas com o seu trabalho ou em suas } \\
\text { atividades do dia a dia, como por exemplo: fez menos do } \\
\text { que gostaria, por casa de sua saúde física. }\end{array}$} \\
\hline Sim & 377 & 51,40 & 51,00 & 2,9 & 2,777 \\
\hline Não & 357 & 48,60 & 49,00 & & \\
\hline \multicolumn{6}{|c|}{$\begin{array}{l}5 \text { - Durante as últimas } 4 \text { semanas, o (a) Sr.(a) teve algum } \\
\text { dos seguintes problemas com o seu trabalho ou em suas } \\
\text { atividades do dia a dia, como por exemplo: sentiu-se com } \\
\text { dificuldade no trabalho ou em outras atividades, por } \\
\text { causa de sua saúde física.* }\end{array}$} \\
\hline Sim & 366 & 49,90 & 49,60 & 2,9 & 2,776 \\
\hline Não & 368 & 50,10 & 50,40 & & \\
\hline \multicolumn{6}{|c|}{$\begin{array}{l}6 \text { - Durante as últimas } 4 \text { semanas, o (a) Sr.(a) teve algum } \\
\text { dos seguintes problemas com o seu trabalho ou em suas } \\
\text { atividades do dia a dia, como por exemplo: fez menos do } \\
\text { que gostaria, por causa de problemas emocionais.* }\end{array}$} \\
\hline Sim & 244 & 33,30 & 32,50 & 2,5 & 2,336 \\
\hline Não & 489 & 66,70 & 67,50 & & \\
\hline
\end{tabular}

Foi constatado menor comprometimento no domínio físico da qualidade de vida entre idosos do sexo masculino, assim como em estudos pré$\operatorname{vios}^{38,39}$. Homens e mulheres desempenham papéis distintos na sociedade e tendem a ter diferentes necessidades. Os homens são menos propensos a buscar a assistência médica ou a priorizar comportamentos de manutenção da saúde ${ }^{7}$. Sugere-se que a menor procura por serviços de saúde ${ }^{7}$ e a baixa percepção de problemas de saúde entre homens, 
Tabela 2. continuação

\begin{tabular}{|c|c|c|c|c|c|}
\hline \multirow[t]{2}{*}{ Variáveis } & \multirow[b]{2}{*}{$\mathbf{n}$} & \multirow[b]{2}{*}{$\%$} & \multicolumn{3}{|c|}{ Erro padrão } \\
\hline & & & $\%^{a}$ & $\%$ & Deff \\
\hline \multicolumn{6}{|c|}{$\begin{array}{l}7 \text { - Durante as últimas } 4 \text { semanas, o (a) Sr.(a) teve algum } \\
\text { dos seguintes problemas com o seu trabalho ou em suas } \\
\text { atividades do dia a dia, como por exemplo: deixou de } \\
\text { fazer seu trabalho ou outras atividades cuidadosamente, } \\
\text { como de costume, por causa de problemas emocionais. }\end{array}$} \\
\hline Sim & 219 & 29,80 & 28,50 & 2,2 & 1,956 \\
\hline Não & 515 & 70,20 & 71,50 & & \\
\hline \multicolumn{6}{|c|}{$\begin{array}{l}8 \text { - Durante as últimas } 4 \text { semanas, alguma dor atrapalhou } \\
\text { seu trabalho normal (tanto o trabalho de casa como o de } \\
\text { fora de casa). }\end{array}$} \\
\hline Extremamente & 19 & 2,60 & 2,60 & 0,8 & 2,182 \\
\hline Bastante & 97 & 13,20 & 13,60 & 1,8 & 2,362 \\
\hline Moderadamente & 109 & 14,90 & 15,00 & 2,1 & 2,882 \\
\hline Um pouco & 189 & 25,70 & 2,90 & 3,4 & 4,788 \\
\hline Nenhum pouco & 320 & 43,60 & 41,90 & 3,1 & 3,187 \\
\hline \multicolumn{6}{|c|}{9 - Quanto tempo durante as últimas 4 semanas o (a) } \\
\hline \multicolumn{6}{|c|}{ Sr.(a) tem se sentido calmo(a) e tranquilo(a).* } \\
\hline Nenhum pouco do tempo & 9 & 1,20 & 0,90 & 0,3 & 1,104 \\
\hline Uma pequena parte do tempo & 71 & 9,70 & 10,40 & 1,7 & 2,449 \\
\hline Alguma parte do tempo & 109 & 14,90 & 15,00 & 1,6 & 1,566 \\
\hline Uma boa parte do tempo & 139 & 18,90 & 19,50 & 2,1 & 2,206 \\
\hline A maior parte do tempo & 335 & 45,60 & 44,80 & 3,9 & 5,126 \\
\hline Todo o tempo & 71 & 9,70 & 9,20 & 1,9 & 3,670 \\
\hline \multicolumn{6}{|c|}{10 - Quanto tempo durante as últimas 4 semanas o (a) } \\
\hline \multicolumn{6}{|c|}{ Sr.(a) teve bastante energia.* } \\
\hline Nenhum pouco do tempo & 10 & 1,30 & 1,00 & 0,4 & 1,189 \\
\hline Uma pequena parte do tempo & 79 & 10,80 & 10,90 & 1,5 & 1,808 \\
\hline Alguma parte do tempo & 124 & 16,90 & 16,40 & 2,3 & 3,208 \\
\hline Uma boa parte do tempo & 190 & 25,90 & 29,70 & 4,5 & 7,865 \\
\hline A maior parte do tempo & 270 & 36,80 & 34,00 & 4,2 & 6,359 \\
\hline Todo o tempo & 61 & 8,30 & 8,00 & 1,7 & 3,327 \\
\hline \multicolumn{6}{|c|}{ 11- Quanto tempo durante as últimas 4 semanas o (a) } \\
\hline \multicolumn{6}{|c|}{ Sr.(a) sentiu-se desanimado(a) e deprimido (a).* } \\
\hline Todo o tempo & 7 & 1,00 & 0,60 & 0,3 & 1,102 \\
\hline A maior parte do tempo & 37 & 5,00 & 4,30 & 1,0 & 1,866 \\
\hline Uma boa parte do tempo & 45 & 6,10 & 6,60 & 0,9 & 1,183 \\
\hline Alguma parte do tempo & 108 & 14,70 & 14,70 & 2,0 & 2,686 \\
\hline Uma pequena parte do tempo & 300 & 40,80 & 40,60 & 3,0 & 3,103 \\
\hline Nenhuma parte do tempo & 236 & 32,10 & 33,10 & 2,5 & 2,254 \\
\hline \multicolumn{6}{|c|}{$\begin{array}{l}12 \text { - Durante as últimas } 4 \text { semanas, em quanto do } \\
\text { seu tempo a sua saúde ou problemas emocionais } \\
\text { atrapalharam suas atividades sociais, tais como: visitar } \\
\text { amigos, parentes, sair, etc. }\end{array}$} \\
\hline Todo o tempo & 12 & 1,60 & 1,10 & 0,4 & 1,015 \\
\hline A maior parte do tempo & 23 & 3,10 & 2,70 & 0,8 & 1,735 \\
\hline Uma boa parte do tempo & 36 & 4,90 & 4,90 & 0,9 & 1,353 \\
\hline Alguma parte do tempo & 75 & 10,20 & 9,00 & 1,4 & 2,068 \\
\hline Uma pequena parte do tempo & 189 & 25,80 & 25,30 & 3,8 & 6,260 \\
\hline Nenhuma parte do tempo & 398 & 54,40 & 56,90 & 3,3 & 3,638 \\
\hline
\end{tabular}

Fonte: Banco de Dados do SB-MOC, 2008/2009. . *Variáveis que apresentaram "n" inferior a736 devido à perda de informações. 
Tabela 3. Distribuição da frequência da população idosa de Montes Claros/MG, quanto ao OHIP 14, 2008/2009, $\mathrm{n}=736$.

\begin{tabular}{|c|c|c|c|c|c|}
\hline Variáveis & $\mathbf{n}$ & $\%$ & $\%^{\mathrm{a}}$ & Erro padrão & Deff \\
\hline \multicolumn{6}{|l|}{ Prevalência do OHIP Impacto } \\
\hline Não & 589 & 80,00 & 81,60 & \multirow[t]{2}{*}{2,1} & \multirow[t]{2}{*}{2,508} \\
\hline Sim & 147 & 20,00 & 18,40 & & \\
\hline \multicolumn{6}{|l|}{ Dimensão 1 - Limitação funcional ${ }^{*}$} \\
\hline Não & 683 & 93,20 & 94,20 & \multirow[t]{2}{*}{1,1} & \multirow[t]{2}{*}{1,894} \\
\hline Sim & 50 & 6,80 & 5,80 & & \\
\hline \multicolumn{6}{|l|}{ Dimensão 2 - Dor física* } \\
\hline Não & 655 & 89,40 & 91,10 & \multirow[t]{2}{*}{1,5} & \multirow[t]{2}{*}{2,144} \\
\hline Sim & 78 & 10,60 & 8,90 & & \\
\hline \multicolumn{6}{|l|}{ Dimensão 3 - Desconforto psicológico* } \\
\hline Não & 672 & 91,60 & 91,00 & \multirow[t]{2}{*}{1,4} & \multirow[t]{2}{*}{1,934} \\
\hline Sim & 62 & 8,40 & 9,00 & & \\
\hline \multicolumn{6}{|l|}{ Dimensão 4 - Incapacidade física ${ }^{*}$} \\
\hline Não & 683 & 93,10 & 93,40 & \multirow[t]{2}{*}{1,0} & \multirow[t]{2}{*}{1,300} \\
\hline Sim & 51 & 6,90 & 6,60 & & \\
\hline \multicolumn{6}{|l|}{ Dimensão 5 - Incapacidade psicológica* } \\
\hline Não & 689 & 94,00 & 95,00 & \multirow[t]{2}{*}{1,2} & \multirow[t]{2}{*}{2,600} \\
\hline Sim & 44 & 6,00 & 5,00 & & \\
\hline \multicolumn{6}{|l|}{ Dimensão 6 - Incapacidade social ${ }^{*}$} \\
\hline Não & 716 & 97,50 & 97,90 & \multirow[t]{2}{*}{0,7} & \multirow[t]{2}{*}{1,726} \\
\hline Sim & 18 & 2,50 & 2,10 & & \\
\hline \multicolumn{6}{|l|}{ Dimensão 7 - Deficiência* } \\
\hline Não & 712 & 97,00 & 96,50 & 1,0 & 2,357 \\
\hline Sim & 22 & 3,00 & 3,50 & & \\
\hline $\begin{array}{l}1 \text { - Você teve problemas para falar al } \\
\text { de problemas com seus dentes, boca }\end{array}$ & & & & & \\
\hline Nunca & 530 & 72,3 & 72,40 & 4,1 & 6,949 \\
\hline Raramente & 89 & 12,1 & 12,60 & 2,3 & 4,079 \\
\hline Às vezes & 84 & 11,4 & 11,20 & 2,0 & 3,406 \\
\hline Repetidamente & 20 & 2,7 & 2,90 & 0,9 & 2,247 \\
\hline Sempre & 11 & 1,5 & 0,90 & 0,4 & 1,156 \\
\hline $\begin{array}{l}2 \text { - Você sentiu que o sabor dos alim } \\
\text { por causa de problemas com seus de } \\
\text { dentadura? }\end{array}$ & & & & & \\
\hline Nunca & 541 & 73,9 & 72,90 & 5,7 & 13,467 \\
\hline Raramente & 63 & 8,6 & 8,50 & 1,8 & 3,516 \\
\hline Às vezes & 95 & 13,0 & 14,70 & 3,7 & 9,128 \\
\hline Repetidamente & 16 & 2,2 & 2,40 & 0,8 & 2,231 \\
\hline Sempre & 18 & 2,5 & 1,50 & 0,5 & 1,262 \\
\hline 3 - Você já sentiu dores fortes na sua & & & & & \\
\hline Nunca & 520 & 71,0 & 70,30 & 4,0 & 6,127 \\
\hline Raramente & 81 & 11,1 & 13,10 & 3,5 & 8,883 \\
\hline Às vezes & 105 & 14,3 & 13,30 & 1,4 & 1,349 \\
\hline Repetidamente & 15 & 2,0 & 1,80 & 0,6 & 1,529 \\
\hline Sempre & 12 & 1,6 & 1,40 & 0,5 & 1,218 \\
\hline $\begin{array}{l}4 \text { - Você tem sentido incômodo ao ce } \\
\text { por causa de problemas com seus de } \\
\text { dentadura? }\end{array}$ & & & & & \\
\hline Nunca & 421 & 57,4 & 57,70 & 5,1 & 8,532 \\
\hline Raramente & 102 & 13,9 & 16,10 & 4,3 & 11,279 \\
\hline Às vezes & 144 & 19,6 & 19,00 & 1,6 & 1,340 \\
\hline Repetidamente & 39 & 5,3 & 4,20 & 0,8 & 1,338 \\
\hline Sempre & 28 & 3,8 & 3,00 & 0,8 & 1,728 \\
\hline
\end{tabular}


Tabela 3. continuação

$\begin{array}{llllll}\text { Variáveis } & \text { n } & \% & \%^{\mathrm{a}} & \text { Erro padrão } & \text { Deff }\end{array}$

5 - Você tem ficado preocupado por causa de problemas com seus dentes, sua boca ou dentadura?

\section{Nunca}

Raramente

Às vezes

Repetidamente

Sempre

6 - Você se sentiu estressado por causa de problemas com seus dentes, sua boca ou dentadura?

Nunca

Raramente

Às vezes

Repetidamente

Sempre

7 - Sua alimentação tem sido prejudicada por causa de problemas com seus dentes, sua boca ou detadura?*

Nunca

Raramente

Às vezes

Repetidamente

Sempre

8 - Você teve que parar suas refeições por causa de seus dentes, sua boca ou dentadura?*

Nunca

Raramente

Às vezes

Repetidamente

Sempre

9 - Você tem tido dificuldades de relaxar por causa de problemas com seus dentes, sua boca ou dentadura?

\begin{tabular}{|c|c|c|c|c|c|}
\hline Nunca & 590 & 80,5 & 78,60 & 6,1 & 18,091 \\
\hline Raramente & 67 & 9,1 & 11,70 & 4,9 & 18,607 \\
\hline Às vezes & 62 & 8,5 & 7,90 & 1,8 & 3,601 \\
\hline Repetidamente & 10 & 1,4 & 1,20 & 0,5 & 1,708 \\
\hline Sempre & 4 & 0,5 & 0,60 & 0,4 & 1,877 \\
\hline \multicolumn{6}{|c|}{$\begin{array}{l}0 \text { - Você já se sentiu um pouco envergonhado por causa de } \\
\text { roblemas com seus dentes, sua boca ou dentadura?* }\end{array}$} \\
\hline Nunca & 531 & 72,3 & 71,40 & 5,5 & 12,152 \\
\hline Raramente & 60 & 8,2 & 9,00 & 1,9 & 3,709 \\
\hline Às vezes & 105 & 14,3 & 15,40 & 4,1 & 10,282 \\
\hline Repetidamente & 13 & 1,8 & 1,50 & 0,5 & 1,452 \\
\hline Sempre & 25 & 3,4 & 2,70 & 0,8 & 2,056 \\
\hline
\end{tabular}

continua

podem influenciar a percepção dos mesmos sobre sua saúde. Assim, eles podem não perceber sua qualidade de vida comprometida, mesmo em situações nas quais tal comprometimento possa existir. Ressalte-se também a dupla jornada de trabalho entre as mulheres, que dividem suas tarefas entre aquelas referentes ao seu trabalho e ao seu papel familiar, gerando cansaço físico ${ }^{40}$, situação

$\begin{array}{rrrrr}459 & 62,5 & 61,30 & 4,8 & 8,044 \\ 88 & 12,0 & 13,40 & 2,9 & 5,984 \\ 130 & 17,7 & 17,10 & 2,4 & 3,350 \\ 27 & 3,7 & 4,00 & 0,9 & 1,901 \\ 30 & 4,1 & 4,30 & 1,3 & 3,140\end{array}$

$\begin{array}{lllll}572 & 78,0 & 76,10 & 3,5 & 5,484\end{array}$

$\begin{array}{lllll}67 & 9,1 & 11,00 & 2,7 & 6,223\end{array}$

$\begin{array}{lllll}69 & 9,4 & 8,70 & 1,3 & 1,746\end{array}$

$\begin{array}{lllll}11 & 1,5 & 1,90 & 0,8 & 2,890\end{array}$

$15 \quad 2,0 \quad 2,30 \quad 0,7 \quad 1,863$

$\begin{array}{rrrrr}489 & 66,6 & 66,10 & 4,9 & 8,778 \\ 85 & 11,6 & 13,20 & 3,0 & 6,321 \\ 113 & 15,4 & 14,30 & 2,3 & 3,367 \\ 30 & 4,1 & 4,20 & 0,9 & 1,723 \\ 17 & 2,3 & 2,10 & 0,7 & 1,910\end{array}$

$\begin{array}{lllll}598 & 81,5 & 82,60 & 2,1 & 2,450\end{array}$

$\begin{array}{lllll}60 & 8,2 & 8,00 & 1,3 & 1,869\end{array}$

$\begin{array}{lllll}59 & 8,0 & 7,30 & 1,2 & 1,875\end{array}$

$\begin{array}{lllll}15 & 2,0 & 2,00 & 0,8 & 2,607\end{array}$

$\begin{array}{rrrrr}15 & 0,3 & 0,20 & 0,1 & 0,754\end{array}$


Tabela 3. continuação

\begin{tabular}{|c|c|c|c|c|c|}
\hline Variáveis & $\mathbf{n}$ & $\%$ & $\%^{\mathrm{a}}$ & Erro padrão & Deff \\
\hline \multicolumn{6}{|c|}{$\begin{array}{l}11 \text { - Você tem estado um pouco irritado com outras pessoas } \\
\text { por problemas com seus dentes, sua boca ou dentadura?* }\end{array}$} \\
\hline Nunca & 649 & 88,4 & 89,40 & 1,6 & 2,203 \\
\hline Raramente & 30 & 4,1 & 3,80 & 0,8 & 1,416 \\
\hline Às vezes & 44 & 6,0 & 5,60 & 1,1 & 1,927 \\
\hline Repetidamente & 8 & 1,1 & 0,80 & 0,3 & 0,988 \\
\hline Sempre & 3 & 0,4 & 0,40 & 0,2 & 1,179 \\
\hline \multicolumn{6}{|c|}{$\begin{array}{l}12 \text { - Você tem dificuldade em realizar suas atividades diárias } \\
\text { por causa de problemas com seus dentes, sua boca ou } \\
\text { dentadura?* }\end{array}$} \\
\hline Nunca & 635 & 86,5 & 85,60 & 3,6 & 8,475 \\
\hline Raramente & 49 & 6,7 & 8,00 & 2,9 & 9,162 \\
\hline Às vezes & 38 & 5,2 & 4,80 & 1,1 & 2,138 \\
\hline Repetidamente & 8 & 1,1 & 1,30 & 0,6 & 2,016 \\
\hline Sempre & 4 & 0,5 & 0,40 & 0,2 & 0,745 \\
\hline \multicolumn{6}{|c|}{$\begin{array}{l}13 \text { - Você já sentiu que a vida em geral ficou pior por causa } \\
\text { de problemas com seus dentes, sua boca ou dentadura? }\end{array}$} \\
\hline Nunca & 633 & 86,3 & 86,10 & 2,4 & 3,800 \\
\hline Raramente & 28 & 3,8 & 4,60 & 1,9 & 6,523 \\
\hline Às vezes & 51 & 6,9 & 5,80 & 1,2 & 2,153 \\
\hline Repetidamente & 8 & 1,1 & 1,30 & 0,6 & 2,044 \\
\hline Sempre & 14 & 1,9 & 2,10 & 0,7 & 1,820 \\
\hline \multicolumn{6}{|c|}{$\begin{array}{l}14 \text { - Você tem estado sem poder fazer suas atividades } \\
\text { por causa de problemas com seus dentes, sua boca ou } \\
\text { dentadura?* }\end{array}$} \\
\hline Nunca & 688 & 93,7 & 92,90 & 1,9 & 4,656 \\
\hline Raramente & 18 & 2,5 & 3,00 & 1,1 & 3,561 \\
\hline Às vezes & 23 & 3,1 & 3,00 & 0,9 & 2,317 \\
\hline Repetidamente & 3 & 0,4 & 0,90 & 0,7 & 4,831 \\
\hline Sempre & 2 & 0,3 & 0,20 & 0,2 & 0,896 \\
\hline
\end{tabular}

"Variáveis que apresentaram "n" inferior a 736 devido à perda de informações.

limitações funcionais e sociais na vida diária dos indivíduos ${ }^{41}$, ou seja, no domínio físico da qualidade de vida. Assim como as doenças crônicas podem interferir na qualidade de vida, a ingestão de bebida alcoólica que pode causar tais doenças ${ }^{42}$ também foi associada a um maior comprometimento do domínio físico da qualidade de vida. A ingestão de bebida alcoólica favorece a ocorrência de problemas sistêmicos, compromete aspectos funcionais, psicológicos e pode ainda evoluir para o óbito. Tal constatação explica a existência da associação entre esse hábito e o comprometimento da qualidade de vida. De forma controversa, em função da possibilidade de causalidade reversa nestas associações, estudo prévio identificou que idosos que não ingeriam bebida alcoólica pelo menos uma vez por semana apresentaram pior qualidade de vida do que os que ingeriam ${ }^{43}$.

Quanto ao serviço de saúde utilizado, o comprometimento da qualidade de vida no domínio físico foi maior nos idosos usuários do SUS. Desde 1988, o Brasil tem estabelecido um sistema de saúde dinâmico e complexo (SUS), baseado nos princípios da saúde como um direito do cidadão e um dever do Estado. O SUS melhorou o acesso da população à atenção primária à saúde, entretanto, alguns desafios ainda precisam ser enfrentados, tais como a reforma de sua estrutura e financiamento, com o intuito de assegurar a universalidade, igualdade, integralidade e a qualidade da assistência. As pessoas que têm acesso a planos ou a seguros de saúde privados também utilizam os serviços disponibilizados pelo SUS, tais como vacinas e serviços de alto custo (procedimentos complexos) ${ }^{31}$, entretanto, parece que o acesso a serviços de saúde, especialmente os preventivos, é maior entre elas do que entre aquelas que não dispõem de planos ou seguros de saúde. Sugere-se que melhorias no SUS, quanto ao acesso, à estrutura e à liberdade na escolha do profissional, entre outros fatores, 
Tabela 4. Análise univariada dos fatores associados ao impacto na qualidade de vida dos idosos de Montes Claros (MG), quanto aos domínios físico e mental, 2008/2009.

\begin{tabular}{|c|c|c|c|c|c|c|c|c|}
\hline \multirow[b]{2}{*}{ Variáveis } & \multicolumn{4}{|c|}{ Domínio físico } & \multicolumn{4}{|c|}{ Domínio mental } \\
\hline & Sim & OR & IC $95 \%$ & $\mathrm{p}$ & Sim & OR & IC 95\% & $\mathrm{p}$ \\
\hline Gravidade OHIP & - & 1,05 & $1,02-1,07$ & 0,001 & - & 1,08 & $1,06-1,11$ & 0,000 \\
\hline Extensão OHIP & - & 1,31 & $1,05-1,64$ & 0,016 & - & 1,55 & $1,29-1,85$ & 0,000 \\
\hline \multicolumn{9}{|l|}{ Prevalência do OHIP Impacto } \\
\hline Não & 61,90 & 1,00 & & & 13,10 & 1,00 & & \\
\hline Sim & 75,60 & 1,90 & $1,11-3,25$ & 0,019 & 42,10 & 4,82 & $2,99-7,79$ & 0,000 \\
\hline \multicolumn{9}{|l|}{ Dimensão 1 - Limitação funcional ${ }^{*}$} \\
\hline Não & 63,00 & 1,00 & & & 16,00 & 1,00 & & \\
\hline Sim & 88,60 & 4,59 & $1,66-12,68$ & 0,002 & 58,10 & 7,26 & $2,98-17,60$ & 0,000 \\
\hline \multicolumn{9}{|l|}{ Dimensão 2 - Dor física ${ }^{*}$} \\
\hline Não & 63,10 & 1,00 & & & 16,40 & 1,00 & & \\
\hline Sim & 78,40 & 2,12 & $1,01-4,45$ & 0,041 & 40,20 & 3,41 & $1,96-5,94$ & 0,000 \\
\hline \multicolumn{9}{|l|}{ Dimensão 3 - Desconforto psicológico* } \\
\hline Não & 63,20 & 1,00 & & & 15,20 & 1,00 & & \\
\hline Sim & 77,40 & 2,00 & $1,01-3,92$ & 0,041 & 51,90 & 6,02 & $3,22-11,25$ & 0,000 \\
\hline \multicolumn{9}{|l|}{ Dimensão 4 - Incapacidade física ${ }^{*}$} \\
\hline Não & 63,2 & 1,00 & & & 16,40 & 1,00 & & \\
\hline Sim & 82,8 & 2,81 & $1,06-7,42$ & 0,031 & 48,20 & 4,72 & $1,95-11,45$ & 0,000 \\
\hline \multicolumn{9}{|l|}{ Dimensão 5 - Incapacidade psicológica* } \\
\hline Não & 64,00 & 1,00 & & & 17,00 & 1,00 & & \\
\hline Sim & 71,80 & 1,42 & $0,53-3,80$ & 0,465 & 48,30 & 4,57 & $1,72-12,17$ & 0,002 \\
\hline \multicolumn{9}{|l|}{ Dimensão 6 - Incapacidade social ${ }^{*}$} \\
\hline Não & 63,90 & 1,00 & & & 17,60 & 1,00 & & \\
\hline Sim & 91,30 & 5,95 & $1,24-28,42$ & 0,012 & 61,80 & 7,57 & $2,29-25,06$ & 0,000 \\
\hline \multicolumn{9}{|l|}{ Dimensão 7 - Deficiência* } \\
\hline Não & 63,60 & 1,00 & & & 16,40 & 1,00 & & \\
\hline Sim & 89,50 & 4,88 & $1,26-18,89$ & 0,013 & 76,50 & 16,58 & $5,04-54,49$ & 0,000 \\
\hline \multicolumn{9}{|l|}{ Características pessoais } \\
\hline \multicolumn{9}{|l|}{ Faixa etária (em anos) } \\
\hline 65 a 68 & 61,70 & 1,00 & & & 17,10 & 1,00 & & \\
\hline 69 a 74 & 67,50 & 1,29 & $0,91-1,81$ & 0,139 & 20,00 & 1,20 & $0,83-1,74$ & 0,311 \\
\hline \multicolumn{9}{|l|}{ Raça autodeclarada } \\
\hline Branco & 67,70 & 1,00 & & & 17,90 & 1,00 & & \\
\hline Pardo/Amarelo/Negro/Indígena & 62,90 & 0,81 & $0,50-1,29$ & 0,373 & 18,70 & 1,05 & $0,64-1,71$ & 0,831 \\
\hline \multicolumn{9}{|l|}{ Sexo } \\
\hline Feminino & 68,40 & 1,00 & & & 22,00 & 1,00 & & \\
\hline Masculino & 59,40 & 0,67 & $0,47-0,96$ & 0,030 & 14,00 & 0,57 & $0,36-0,92$ & 0,022 \\
\hline \multicolumn{9}{|l|}{ Estado Civil } \\
\hline Casado/União estável & 61,60 & 1,00 & & & 17,90 & 1,00 & & \\
\hline Viúvo/Divorciado & 70,90 & 1,52 & $0,97-2,36$ & 0,063 & 18,10 & 1,01 & $0,70-1,46$ & 0,945 \\
\hline Solteiro & 61,10 & 0,97 & $0,47-2,01$ & 0,948 & 30,30 & 1,99 & $0,76-5,21$ & 0,154 \\
\hline \multicolumn{9}{|l|}{ Escolaridade (anos de estudo) } \\
\hline 9 ou mais anos & 53,20 & 1,00 & & & 14,80 & 1,00 & & \\
\hline 5 a 8 anos & 57,60 & 1,19 & $0,69-2,06$ & 0,504 & 16,50 & 1,13 & $0,53-2,43$ & 0,736 \\
\hline 0 a 4 anos & 68,20 & 1,88 & $0,98-3,61$ & 0,054 & 19,70 & 1,41 & $0,72-2,76$ & 0,302 \\
\hline \multicolumn{9}{|l|}{ Renda per capita em salários mínimos ${ }^{\mathrm{b}}$} \\
\hline Mais de 1 salário & 63,20 & 1,00 & & & 18,50 & 1,00 & & \\
\hline Até 1 salário & 66,00 & 1,13 & $0,78-1,63$ & 0,495 & 19,50 & 1,06 & $0,64-1,76$ & 0,799 \\
\hline
\end{tabular}

podem gerar maior satisfação com tais serviços e resolução dos problemas de saúde, o que, consequentemente, pode gerar menor comprometi- mento da qualidade de vida. Além disso, algumas limitações funcionais e sistêmicas são inerentes ao envelhecimento. Acredita-se que o uso regular 
Tabela 4. continuação

\begin{tabular}{|c|c|c|c|c|c|c|c|c|}
\hline \multirow[b]{2}{*}{ Variáveis } & \multicolumn{4}{|c|}{ Domínio físico } & \multicolumn{4}{|c|}{ Domínio mental } \\
\hline & Sim & OR & IC $95 \%$ & $\mathbf{p}$ & Sim & OR & IC 95\% & p \\
\hline \multirow{2}{*}{\multicolumn{9}{|c|}{$\begin{array}{l}\text { Condições subjetivas e normativas de saúde geral } \\
\text { Presença de doenças crônicas }\end{array}$}} \\
\hline & & & & & & & & \\
\hline Não & 37,10 & 1,00 & & & 12,10 & 1,00 & & \\
\hline Sim & 70,20 & 4,00 & $2,34-6,85$ & 0,000 & 19,90 & 1,80 & $0,84-3,83$ & 0,118 \\
\hline \multicolumn{9}{|l|}{ Uso de medicamentos } \\
\hline Não & 49,80 & 1,00 & & & 11,10 & 1,00 & & \\
\hline Sim & 70,00 & 2,35 & $1,58-3,48$ & 0,000 & 21,30 & 2,16 & $1,27-3,65$ & 0,004 \\
\hline \multicolumn{9}{|c|}{ Mini-Mental (ajustado por escolaridade) } \\
\hline Sem déficit cognitivo & 59,20 & 1,00 & & & 17,60 & 1,00 & & \\
\hline Com déficit cognitivo & 74,30 & 1,99 & $1,15-3,45$ & 0,014 & 20,50 & 1,20 & $0,68-2,11$ & 0,502 \\
\hline \multicolumn{9}{|l|}{ Atividade física } \\
\hline Sempre ou frequentemente & 59,70 & 1,00 & & & 15,60 & 1,00 & & \\
\hline Ocasionalmente & 67,90 & 1,42 & $0,77-2,63$ & 0,251 & 20,40 & 1,38 & $0,71-2,65$ & 0,325 \\
\hline Raramente ou nunca & 64,90 & 1,25 & $0,71-2,19$ & 0,427 & 18,90 & 1,25 & $0,70-2,21$ & 0,428 \\
\hline \multicolumn{9}{|l|}{ Satisfação com a vida } \\
\hline Satisfeito ou muito satisfeito & 61,10 & 1,00 & & & 13,80 & 1,00 & & \\
\hline Nem satisfeito e nem insatisfeito & 84,70 & 3,52 & $1,59-7,82$ & 0,003 & 37,80 & 3,80 & $2,30-6,29$ & 0,000 \\
\hline Insatisfeito ou muito insatisfeito & 59,90 & 0,95 & $0,42-2,13$ & 0,898 & 54,60 & 7,52 & $2,94-19,27$ & 0,000 \\
\hline \multicolumn{9}{|l|}{ Comportamentos } \\
\hline \multicolumn{9}{|l|}{ Higiene bucal } \\
\hline \multicolumn{9}{|l|}{ Frequência diária da higiene bucal } \\
\hline Mais de 2 vezes & 63,50 & 1,00 & & & 17,20 & 1,00 & & \\
\hline 1 ou 2 vezes & 64,50 & 1,04 & $0,66-1,64$ & 0,844 & 19,20 & 1,14 & $0,72-1,82$ & 0,549 \\
\hline Nenhuma vez & 65,60 & 1,09 & $0,50-2,35$ & 0,813 & 8,30 & 0,43 & $0,11-1,71$ & 0,228 \\
\hline \multicolumn{9}{|l|}{ Hábito tabagista atual ou passado } \\
\hline Ausente & 63,00 & 1,00 & & & 17,60 & 1,00 & & \\
\hline Presente & 67,20 & 1,20 & $0,77-1,85$ & 0,400 & 20,30 & 1,19 & $0,76-1,86$ & 0,427 \\
\hline \multicolumn{9}{|l|}{ Hábito etilista atual ou passado } \\
\hline Ausente & 61,00 & 1,00 & & & 17,30 & 1,00 & & \\
\hline Presente & 69,70 & 1,46 & $0,96-2,23$ & 0,069 & 20,40 & 1,22 & $0,73-2,05$ & 0,430 \\
\hline \multicolumn{9}{|l|}{ Serviço odontológico utilizado } \\
\hline Outros serviços & 72,00 & 1,00 & & & 18,40 & 1,00 & & \\
\hline SUS & 61,10 & 1,63 & $1,00-2,65$ & 0,047 & 18,00 & 1,03 & $0,64-1,65$ & 0,892 \\
\hline \multicolumn{9}{|l|}{ Condições normativas de Saúde Bucal } \\
\hline \multicolumn{9}{|l|}{ Alterações da mucosa bucal } \\
\hline Não & 60,80 & 1,00 & & & 16,00 & 1,00 & & \\
\hline Sim & 76,30 & 2,07 & $1,24-3,47$ & 0,006 & 27,00 & 1,95 & $1,23-3,08$ & 0,005 \\
\hline \multicolumn{9}{|l|}{ Presença de edentulismo } \\
\hline Não & 63,60 & 1,00 & & & 18,80 & 1,00 & & \\
\hline Sim & 65,00 & 1,06 & $0,74-1,51$ & 0,723 & 18,30 & 0,97 & $0,65-1,43$ & 0,886 \\
\hline \multicolumn{9}{|l|}{ Uso de prótese dentária } \\
\hline Não & 66,30 & 1,00 & & & 19,10 & 1,00 & & \\
\hline Sim & 63,90 & 0,90 & $0,61-1,30$ & 0,572 & 18,30 & 0,95 & $0,59-1,51$ & 0,833 \\
\hline \multicolumn{9}{|l|}{$\begin{array}{l}\text { Necessidade normativa de tratamento } \\
\text { odontológico }\end{array}$} \\
\hline Não & 57,60 & 1,00 & & & 16,40 & 1,00 & & \\
\hline Sim & 67,10 & 1,49 & $0,81-2,74$ & 0,185 & 19,30 & 1,22 & $0,73-2,03$ & 0,427 \\
\hline
\end{tabular}

de serviços de saúde disponibilizados pelo SUS e maior atenção à saúde do idoso poderiam minimizar os efeitos dessas limitações. Contudo, este contingente idoso parece ter encontrado dificuldades no acesso regular ao serviço odontológico, uma vez que 5,8\% dos idosos brasileiros ${ }^{44}$ e 1,6\% 
Tabela 4. continuação

\begin{tabular}{|c|c|c|c|c|c|c|c|c|}
\hline \multirow[b]{2}{*}{ Variáveis } & \multicolumn{4}{|c|}{ Domínio físico } & \multicolumn{4}{|c|}{ Domínio mental } \\
\hline & Sim & OR & IC 95\% & $\mathbf{p}$ & Sim & OR & IC 95\% & p \\
\hline \multicolumn{9}{|c|}{ Condições subjetivas de Saúde Bucal } \\
\hline \multicolumn{9}{|l|}{ Percepção da saúde bucal } \\
\hline Ótima ou boa & 60,30 & 1,00 & & & 14,10 & 1,00 & & \\
\hline Regular & 71,70 & 1,67 & $1,14-2,45$ & 0,010 & 23,30 & 1,84 & $1,10-3,08$ & 0,020 \\
\hline Ruim ou péssima & 77,80 & 2,31 & $0,94-5,68$ & 0,065 & 47,00 & 5,38 & $2,28-12,67$ & 0,000 \\
\hline \multicolumn{9}{|l|}{ Percepção da mastigação } \\
\hline Ótima ou boa & 62,90 & 1,00 & & & 16,30 & 1,00 & & \\
\hline Regular & 67,20 & 1,20 & $0,74-1,95$ & 0,435 & 18,70 & 1,18 & $0,69-2,00$ & 0,526 \\
\hline Ruim ou péssima & 65,30 & 1,11 & $0,59-2,08$ & 0,740 & 27,20 & 1,92 & $0,91-4,05$ & 0,084 \\
\hline \multicolumn{9}{|c|}{ Percepção da aparência de dentes e gengivas } \\
\hline Ótima ou boa & 61,60 & 1,00 & & & 15,10 & 1,00 & & \\
\hline Regular & 67,50 & 1,29 & $0,84-1,97$ & 0,226 & 20,20 & 1,42 & $0,85-2,35$ & 0,168 \\
\hline Ruim ou péssima & 70,10 & 1,46 & $0,70-3,03$ & 0,299 & 30,50 & 2,46 & $1,03-5,85$ & 1,042 \\
\hline \multicolumn{9}{|c|}{ Percepção da fala devido aos dentes e gengivas } \\
\hline Ótima ou boa & 62,60 & 1,00 & & & 16,00 & 1,00 & & \\
\hline Regular & 67,40 & 1,23 & $0,68-2,21$ & 0,470 & 19,70 & 1,28 & $0,57-2,88$ & 0,534 \\
\hline Ruim ou péssima & 81,80 & 2,67 & $1,14-6,28$ & 0,025 & 50,30 & 5,29 & $2,27-12,33$ & 0,000 \\
\hline \multicolumn{9}{|c|}{$\begin{array}{l}\text { Percepção do relacionamento devido à } \\
\text { condição bucal }\end{array}$} \\
\hline Não afetado & 63,70 & 1,00 & & & 16,00 & 1,00 & & \\
\hline Afetado & 65,90 & 1,10 & $0,64-1,87$ & 0,716 & 23,50 & 1,61 & $1,12-2,31$ & 0,010 \\
\hline \multicolumn{9}{|c|}{$\begin{array}{l}\text { Percepção da necessidade de tratamento } \\
\text { odontológico }\end{array}$} \\
\hline Sim & 61,90 & 1,00 & & & 16,90 & 1,00 & & \\
\hline Não & 67,60 & 1,28 & $0,78-2,11$ & 0,306 & 20,30 & 1,24 & $0,75-2,07$ & 0,380 \\
\hline \multicolumn{9}{|c|}{ Dor em dentes e gengivas nos últimos 6 meses } \\
\hline Não & 61,40 & 1,00 & & & 17,10 & 1,00 & & \\
\hline $\operatorname{Sim}$ & 74,50 & 1,83 & $1,22-2,75$ & 0,004 & 23,40 & 1,47 & $0,87-2,48$ & 0,137 \\
\hline \multicolumn{9}{|c|}{ Satisfação com atendimento odontológico } \\
\hline Satisfeito & 64,30 & 1,00 & & & 17,40 & 1,00 & & \\
\hline Insatisfeito & 75,60 & 1,71 & $0,87-3,37$ & 0,110 & 24,40 & 1,53 & $0,70-3,32$ & 0,267 \\
\hline
\end{tabular}

a Baseada no salário mínimo em 2008, no valor de $\mathrm{R} \$ 415,00$. Algumas variáveis apresentaram $\mathrm{n}<736$ devido à perda de informações.

dos idosos de Montes Claros relataram nunca ter utilizado tais serviços.

Entre as condições subjetivas de saúde bucal, observou-se maior comprometimento do domínio físico em quem autopercebeu a presença de dor em dentes e gengivas nos últimos seis meses. Em investigação prévia, a presença de dor crônica foi associada a um maior comprometimento do domínio físico da qualidade de vida ${ }^{45}$, constatando-se a influência da dor na qualidade de vida. A autopercepção da dor em dentes e gengivas pode refletir as precárias condições de saúde bucal dos idosos brasileiros ${ }^{37}$ e montes-clarenses. Essa dor em dentes e gengivas pode gerar dificuldades no exercício das funções bucais corretamente, com- prometendo, assim, o domínio físico da qualidade de vida.

Tradicionalmente no campo da saúde, como consequência da herança do modelo biomédico de saúde ${ }^{46}$, valorizou-se os dados referentes às condições clínicas da doença em si. Entretanto, medidas de saúde devem levar em consideração as influências sociais, ambientais e psicológicas ${ }^{18}$. Nesse contexto, constatou-se maior comprometimento do domínio mental da qualidade de vida entre idosos que apresentaram maior gravidade do OHIP, além de impacto nas dimensões referentes ao desconforto psicológico e deficiência. Portanto, os impactos das desordens bucais em suas dimensões física e psicossocial estiveram associados a 
Tabela 5. Análise multivariada dos fatores associados ao comprometimento da qualidade de vida dos idosos de Montes Claros (MG), 2008/2009.

\begin{tabular}{|c|c|c|c|c|}
\hline \multirow[b]{2}{*}{ Condição avaliada } & \multicolumn{2}{|c|}{ Domínio físico } & \multicolumn{2}{|c|}{ Domínio mental } \\
\hline & OR (IC95\%) & $\mathbf{p}$ & OR (IC95\%) & p \\
\hline Gravidade OHIP & - & & $1,06(1,03-1,09)$ & 0,000 \\
\hline \multicolumn{5}{|l|}{ Dimensão 1 - Limitação funcional } \\
\hline Não & 1 & - & - & - \\
\hline Sim & $3,14(1,12-8,77)$ & 0,030 & - & - \\
\hline \multicolumn{5}{|l|}{ Dimensão 3 - Desconforto psicológico } \\
\hline Não & - & - & 1 & - \\
\hline Sim & - & - & $2,23(0,99-5,02)$ & 0,051 \\
\hline \multicolumn{5}{|l|}{ Dimensão 7 - Deficiência } \\
\hline Não & - & - & 1 & - \\
\hline Sim & - & - & $6,79(1,34-34,40)$ & 0,022 \\
\hline \multicolumn{5}{|l|}{ Características pessoais } \\
\hline \multicolumn{5}{|l|}{ Sexo } \\
\hline Feminino & 1 & - & - & - \\
\hline Masculino & $0,66(0,46-0,95)$ & 0,027 & - & - \\
\hline \multicolumn{5}{|c|}{ Condições subjetivas e normativas de saúde geral } \\
\hline \multicolumn{5}{|l|}{ Presença de doenças crônicas } \\
\hline Não & 1 & - & - & - \\
\hline Sim & $4,41(2,68-7,28)$ & 0,000 & - & - \\
\hline \multicolumn{5}{|l|}{ Uso de medicamentos } \\
\hline Não & - & - & 1 & - \\
\hline Sim & - & - & $2,72(1,43-5,16)$ & 0,003 \\
\hline \multicolumn{5}{|l|}{ Satisfação com a vida } \\
\hline Satisfeito ou muito satisfeito & - & - & 1 & - \\
\hline Nem satisfeito e nem insatisfeito & - & - & $3,56(1,89-6,69)$ & 0,000 \\
\hline Insatisfeito ou muito insatisfeito & - & - & $7,26(2,73-19,27)$ & 0,000 \\
\hline \multicolumn{5}{|l|}{ Comportamentos } \\
\hline \multicolumn{5}{|l|}{ Hábito etilista atual ou passado } \\
\hline Ausente & 1 & - & - & - \\
\hline Presente & $1,92(1,20-3,07)$ & 0,007 & - & - \\
\hline \multicolumn{5}{|l|}{ Serviço odontológico utilizado } \\
\hline Outros serviços & 1 & - & - & - \\
\hline SUS & $1,65(1,01-2,68)$ & 0,043 & - & - \\
\hline \multicolumn{5}{|l|}{ Condições subjetivas de Saúde Bucal } \\
\hline \multicolumn{5}{|c|}{ Dor em dentes e gengivas nos últimos 6 meses } \\
\hline Não & 1 & - & - & - \\
\hline Sim & $1,85(1,17-2,92)$ & 0,009 & - & - \\
\hline
\end{tabular}

não se aplica.

pior qualidade de vida, em seu domínio mental, entre idosos brasileiros. A saúde bucal, um dos componentes da qualidade de vida, refere-se a uma experiência subjetiva do indivíduo sobre o seu bem-estar funcional, social e psicológico ${ }^{47}$.

As dimensões do OHIP que se mantiveram associadas ao domínio mental foram desconforto psicológico e deficiência, era esperado o impacto dessas dimensões nesse domínio da qualidade de vida. Ressalta-se que, apesar de serem questões subjetivas, tais impactos podem estimular a busca por assistência médica ou odontológica ${ }^{9}$, fato este que pode ser ainda mais incentivado quando há o comprometimento da qualidade de vida.

A maioria dos idosos investigados possuía alguma doença crônica e, consequentemente, fazia uso de medicamentos. Sabe-se que o tratamento de diversas patologias requer a utilização de terapia medicamentosa. Os idosos que faziam uso de medicamentos tiveram maior comprometimento no domínio mental da qualidade de vida. Um alto consumo de medicamentos entre 
idosos reside na maior ocorrência de problemas de saúde nas idades mais avançadas, geralmente de longa duração e com maior grau de severidade, cujo tratamento e alívio de sintomas demanda terapia farmacológica ${ }^{48}$. Portanto, o fato de o uso de medicamentos estar ligado à presença de doenças pode comprometer o bem estar dos idosos e consequentemente sua qualidade de vida. Além disso, salienta-se que o uso racional de medicamentos entre idosos pode assegurar boa qualidade de vida a esses indivíduos ${ }^{49}$. Porém, sabe-se que esta adesão entre idosos pode ser comprometida pela falta de conhecimento e dificuldade de acesso ${ }^{50}$. Ressalte-se ainda a possibilidade de causalidade reversa nesta associação.

Os idosos que relataram estar nem satisfeitos/ nem insatisfeitos e insatisfeitos/muito insatisfeitos com sua vida tinham maior comprometimento na qualidade de vida no domínio mental do SF12. A satisfação com a vida na velhice pode estar relacionada à sensação de conforto, bem-estar, saúde, relações pessoais, entre outros fatores ${ }^{51,52}$, podendo ser uma avaliação subjetiva da qualidade de vida. Indivíduos que avaliam sua saúde bucal como ruim, talvez devido à presença de problemas bucais, podem apresentar maior insatisfação com sua vida ${ }^{53}$, o que pode influenciar na qualidade de vida. Acredita-se que a satisfação com a vida, por ser uma questão subjetiva, é um julgamento cognitivo de alguns domínios específicos da vida, a partir de critérios estabelecidos pelo próprio indivíduo, sendo uma questão psicológica, podendo afetar o domínio mental da qualidade de vida. Além disso, considera-se também a possibilidade de causalidade reversa nesta associação.

Dentre as limitações dessa investigação, ressalta-se sua característica transversal, não sendo possível estabelecer relações de causa e feito entre os fatores associados. Apesar disso, os resultados apresentados são válidos e representativos da população idosa investigada, situação que permitiu mais esclarecimentos sobre a qualidade de vida desses idosos. Salienta-se ainda que a avaliação da qualidade de vida é um processo dinâmico, e possíveis fatores associados podem modificar-se com o passar do tempo.

\section{Conclusão}

O OHIP avalia, a partir de questões subjetivas, os impactos decorrentes das desordens bucais, já o SF12 avalia a qualidade de vida. Constatou-se que o OHIP e o SF12 avaliam construtos distintos embora possam estar associados. A gravidade desses impactos, as dimensões referentes à limitação funcional, ao desconforto psicológico e à deficiência foram associadas a uma maior chance de comprometimento na qualidade de vida em seus domínios físico e mental. Os impactos decorrentes das desordens bucais devem ser considerados nas políticas de saúde que têm em vista a qualidade de vida dos idosos.

\section{Colaboradores}

AMEBL Martins participou da concepção do projeto, análise e interpretação dos dados e redação do artigo. JGS Souza e KM Jones participaram da análise e interpretação dos dados e redação do artigo. IA Pordeus participou da análise e interpretação dos dados, redação do artigo, revisão crítica relevante do conteúdo intelectual e aprovação final da versão a ser publicada.

\section{Agradecimentos}

Agradecimentos ao apoio logístico da Unimontes e da Prefeitura Municipal de Montes Claros-MG, ao fomento da Fundação de Amparo a Pesquisa do Estado de Minas Gerais-FAPEMIG e a colaboração dos participantes. As pesquisadoras Andréa Maria Eleutério de Barros Lima Martins e Isabela Almeida Pordeus são bolsistas do CNPq. 


\section{Referências}

1. Kandelman D, Petersen PE, Ueda H. Oral health, general health, and quality of life in older people. Spec Care Dentist 2008; 28(6):224-236.

2. Christensen K, Doblhammer G, Rau R, Vaupel JW. Ageing populations: the challenges ahead. Lancet 2009; 374(9696):1196-1208.

3. The WHOQOL Group. The World Health Organization quality of life assessment (WHOQOL): position paper from the World Health Organization. Soc Sci Med 1995; 41(10):1403-1409.

4. López-Jornet P, Camacho-Alonso F, Lucero-Berdugo M. Quality of life in patients with burning mouth syndrome. J Oral Pathol Med 2008; 37(7):389-394.

5. Oliva-Moreno J, Lopez-Bastida J, Worbes-Cerezo M, Serrano-Aguilar P. Health related quality of life of Canary Island citizens. BMC Public Health 2010; 10:675.

6. McGorrian C, McShane C, McQuade C, Keelan T, Neill JO, Galvin J, Malone K, Mahon NG, Codd M. Family-based associations in measures of psychological distress and quality of life in a cardiac screening clinic for inheritable cardiac diseases: a cross-sectional study. BMC Medical Genetics 2013; 14:1.

7. Solimeo SL, Silverman SL, Calderon AD, Nguyen A, Gold DT. Measuring health-related quality of life (HRQOL) in osteoporotic males using the Male OPAQ. Osteoporos Int 2012; 23(3):841-852.

8. Sheiham A, Steele JG, Marcenes W, Tsakos G, Finch S, and Walls AWG. Prevalence of impacts of dental and oral disorders and their effects on eating among older people; a national survey in Great Britain. Community Dent Oral Epidemiol 2001; 29(3):195-103.

9. Locker D, Quinõnez C. To what extent do oral disorders compromise the quality of life? Community Dent Oral Epidemiol 2011; 39(1):3-11.

10. Tesch FC, Oliveira BH, Leão A. Mensuração do impacto dos problemas bucais sobre a qualidade de vida de crianças: aspectos conceituais e metodológicos. Cad Saude Publica 2007; 23(11):2555-2564.

11. Yiengprugsawan V, Somkotra T. Oral Health-Related Quality of Life among a large national cohort of 87,134 Thai adults. Health Qual Life Outcomes 2011; 9(1):42.

12. Gift HC, Atchison KA, Drury TF. Perceptions of the natural dentition in the context of multiple variables. $J$ Dent Res 1998; 77(7):1529-1538.

13. Ekbäck G, Astrøm AN, Klock K, Ordell S, Unell L. Variation in subjective oral health indicators of 65 -yearolds in Norway and Sweden. Acta Odontol Scand 2009; 67(4):222-232.

14. Reisine ST, Fertig J, Weber J, Leder S. Impact of dental cotiditions on patients' quality of life. Community Dent Oral Epidemiol 1989; 17(1):7-10.

15. Slade GD. Derivation and validation of a short-form oral health impact profile. Community Dent Oral Epidemiol 1997; 25(4):284-290.

16. Andrade FB, Lebrão ML, Santos JL, Cruz Teixeira DS, Oliveira Duarte YA. Relationship between oral health -related quality of life, oral health, socioeconomic, and general health factors in elderly Brazilians. J Am Geriatr Soc 2012; 60(9):1755-1760.

17. Jensen PM, Saunders RL, Thierer T, Friedman B. Factors associated with oral health-related quality of life in community-dwelling elderly persons with disabilities. J Am Geriatr Soc 2008; 56(4):711-717.
18. Allen PF. Assessment of oral health related quality of life. Health Qual Life Outcomes 2003; 1:40.

19. Sanders AE, Slade GD, Lim S, Reisine ST. Impact of oral disease on quality of life in the US and Australian populations. Community Dent Oral Epidemiol 2009; 37(2):171-181.

20. Camelier AA. Avaliação da Qualidade de Vida Relacionada à Saúde em Pacientes com DPOC: estudo de base populacional com o SF-12 na cidade de São Paulo-SP [tese]. São Paulo: Universidade Federal de São Paulo; 2004.

21. Martins AMEBL, Guimarães ALS, De'Paula AMB, Pires CPB, Haikal DAS, Silva JMS, Silveira MF, Caldeira TCR, Eleutério NB, Silveira AM, Almeida BM, Almeida CM, Freitas CV, Botelho DMM, Chaves KTS, Pereira PMB, Lima RS, Pereira SM, Silva TF, Duarte VM, Silva VEP, Pordeus IA. Levantamento epidemiológico das condições de saúde bucal da população de Montes Claros - Projeto SBMOC. RUC 2012; 14(1):3-14.

22. Martins AMEBL, Haikal DAS, Santos-Neto PE, Alves SFF, Eleutério NB, Oliveira PHA, Gomes GP, Guimarães BL, Ferreira RC, Silveira MF, Pordeus IA. Calibração de examinadores do Levantamento epidemiológico das condições de saúde bucal da população de Montes Claros, MG - Projeto SBMOC. RUC 2012; 14(1):43-56.

23. Martins AMEBL, Rodrigues CAQ, Haikal DAS, Silveira MF, Mendes DC, Oliveira MP, Andrade AF, Freitas $\mathrm{CV}$, Pordeus IA. Desenvolvimento de um programa de computador para levantamentos epidemiológicos sobre condições de saúde bucal. RUC 2012; 14(1):30-42.

24. Martins AMEBL, Santos-Neto PE, Batista LHS, Nascimento JE, Gusmão AF, Eleutério NB, Guimarães ALS, Paula AMB, Haikal DS, Silveira MF, Pordeus IA. Plano amostral e ponderação pelo efeito de desenho de um levantamento epidemiológico de saúde bucal. RUC 2012; 14(1):15-29.

25. World Health Organization. Oral health surveys: basic methods. $4^{\text {th }}$ ed. Geneva: ORH/EPID; 1997.

26. Fleiss JL. Statistical methods for rates and proportions. New York: John Wiley Sons; 1981.

27. Cicchetti DV, Volkmar F, Sparrow SS, Cohen D, Fermanian J, Rourke BP. Assessing the reliability of clinical scales when the data have both nominal and ordinal features: proposed guidelines for europsychological assessments. J Clin Exp Neuropsychol 1992; 14(5):673-686.

28. Sanda MG, Wei JT, Litwin MS. Scoring Instructions for the Expanded Prostate cancer Index Composite (EPIC). EPIC [serial on the Internet] 2002 [cited 2013 Jun 5]. [about 5 p.]. Available from: http://www.med.umich. edu/urology/research/EPIC/EPIC-Scoring-2.2002.pdf

29. Oliveira BH, Nadanovsky P. Psychometric properties of the Brasilian version of the oral Health Impact Profile-Short form. Community Dent Oral Epidemiol 2005; 33(4):307-314.

30. Bertolucci PHF, Brucki SMD, Campacci SR, Juliano Y. O mini-exame do estado mental em uma população geral: impacto da escolaridade. Arq Neuro psiquiatr 1994; 52(1):1-7.

31. Paim J, Travassos C, Almeida C, Bahia L, Macinko J. The Brazilian health system: history, advances, and challenges. Lancet 2011; 377(9779):1778-1797. 
32. Brasil. Ministério da Saúde (MS). Conselho Nacional de Saúde. Resolução no. 196 de 10 de outubro de 1996. Diretrizes e Normas Regulamentadoras de Pesquisas Envolvendo Seres Humanos. Diário Oficial da União 1996; 16 out.

33. Slade GD, editor. Measuring Oral Health and Quality of Life. Chapel Hill: University of North Carolina, Dental Ecology; 1997.

34. Xia P, Li N, Hau KT, Liu C, Lu Y. Quality of life of Chinese urban community residents: a psychometric study of the mainland Chinese version of the WHOQOL-BREF. BMC Med Res Methodol 2012; 12:37.

35. Bras M, Milunović V, Boban M, Brajković L, Benković V, Đorđević V, Polašek O. Quality of life in Croatian Homeland war (1991-1995) veterans who suffer from post-traumatic stress disorder and chronic pain. Health Qual Life Outcomes 2011; 9:56.

36. Petersen PE. The World Oral Health Report 2003: Continuous Improvement of Oral Health in the 21st Century - The Approach of the WHO Global Oral Health Programme. Geneva: World Health Organization; 2003.

37. Martins AMEBL, Barreto SM, Silveira MF, Santa-Rosa TTA, Pereira RD. Autopercepção da saúde bucal entre idosos brasileiros. Rev Saude Publica 2010; 44(5):912922.

38. Cruz LN, Polannczky CA, Camey SA, Hoffman JF, Flek MP. Quality of life in Brazil: normative values for the Whoqol-bref in a southern general population sample. Qual Life Res 2011; 20(7):1123-1129.

39. Lee SW, Lien HC, Chang CS, Peng YC, Ko CW, Chou MC. Impact of body mass index and gender on quality of life in patients with gastroesophageal reflux disease. World J Gastroenterol 2012; 18(36):5090-5095.

40. Brito JC. Enfoque de gênero e relação saúde/trabalho no contexto de reestruturação produtiva e precarização do trabalho. Cad Saude Publica 2000; 16(1):195-204.

41. Boing AF, Melo GR, Boing AC, Moretti-Pires RO, Peres KG, Peres MA. Association between depression and chronic diseases: results from a population-based study. Rev Saude Publica 2012; 46(4):617-623.

42. Rehm J, Mathers C, Popova S, Thavorncharoensap M, Teerawattananon Y, Patra J. Global burden of disease and injury and economic cost attributable to alcohol use and alcohol-use disorders. Lancet 2009; 373(9682):22232233.

43. Lima MG, Barros MBA, Cesar CLG, Goldbaum M, Carandina L, Alves MCGP. Health-related behavior and quality of life among the elderly: a population-based study. Rev Saude Publica 2011; 45(3):485-493.
44. Martins AMEBL, Barreto SM, Pordeus IA. Uso de serviços odontológicos entre idosos brasileiros. Rev Panam Salud Publica 2007; 22(5):308-316.

45. Loyland B, Miaskowski C, Paul SM, Dahl E, Rustoen T. The relationship between chronic pain and health-related quality of life in long-term social assistance recipients in Norway. Qual Life Res 2010; 19(10):1457-1465.

46. Mitre SM, Andrade IEG, Cotta RMM. Avanços e desafios do acolhimento na operacionalização e qualificação do Sistema Único de Saúde na Atenção Primária: um resgate da produção bibliográfica do Brasil. Cien Saude Colet 2012; 17(8):2071-2085.

47. Locker D. Clinical correlates of change in self perceived oral health in older adults. Community Dental Oral Epidemiol 1997; 25(3):199-103.

48. Filho AIL, Uchoa E, Lima-Costa MF. A population-based study on use of medication by the elderly in Greater Metropolitan Belo Horizonte, Minas Gerais, Brazil. Cad Saude Publica 2006; 22(12):2657-2667.

49. Nobrega OT, Karnikowski MGO. A terapia medicamentosa no idoso: cuidados na medicação; Cien Saude Colet 2005; 10(2):309-313.

50. Oliveira MPF, Novaes MRCG. Perfil socioeconômico, epidemiológico e farmacoterapêutico de idosos institucionalizados de Brasília, Brasil. Cien Saude Colet 2013; 18(4):1069-1078.

51. Joia LC, Ruiz T, Donalisio MR. Life satisfaction among elderly population in the city of Botucatu, Southern Brazil. Rev Saude Publica 2007; 41(1):131-138.

52. Sposito G, D’Elboux MJ, Neri AL, Guariento ME. A satisfação com a vida e a funcionalidade em idosos atendidos em um ambulatório de geriatria. Cien Saude Colet 2013; 18(12):3475-3482.

53. Locker D, Clarke M, Payne B. Self-perceived Oral Health Status, Psychological Well-being, and Life Satisfaction in an Older Adult Population. J Dent Res 2000; 79(4):970-975.

Artigo apresentado em 04/09/2013

Aprovado em 08/10/2013

Versão final apresentada em 16/10/2013 